unl mit Abstreifen der griech. Lindung *BOT 20P-BOOOP. 1)er $A b f_{a l l}$ der griech. Endung erklïrt sich gewif durch den Gleichklang init dem Gott Horos, den der Kopte sowohl in der einheimischen Form $2 \omega \mathrm{P}$ als uuch in der gräzisierten 'Qgos kannte. Dafi man für den ägypt. Vertreter' die einlreimisch klingende form wihlte, ist sehr natürlich. Aber auch mus *B3O'T2 OP' liebe sich mit Abfall des schwer hinter P жu sprechenden C BOQOP erklären. Aelnliche Konsonantenausfaille sind in den im Kuptischen entlehnten griech. Fremd-

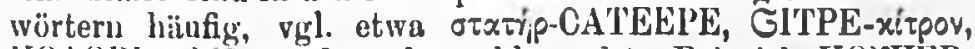

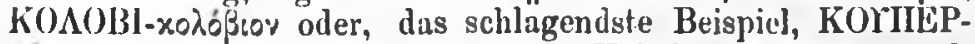
xúmpos, elsenfalls nit Umspringen des Vokals und Abfall des $\mathrm{C}$. Ist somit Bévpos-Bơop identisch ${ }^{8}$ ) und stammen beide aus lom Arabischen, so ist für den Kambysesroman wie fur die oben genannten 'Iralistate ein terminus post quem in der arabischen Eroberung Aegyptens $11 \mathrm{~m} \mathrm{640}$ gegeben ${ }^{\circ}$ ). Dab ein arabischer Astrolog als Schreiber an den Perserkönig ausersehen wurde, ist bei den durchaus verworrenen geschichtlichen Vorstellungen, die der Romansclıreiber zeigt, nicht verwunderlich.

Heidelberg.

Friedrich Bilabel.

\title{
9. Aus der Werkstatt des Athenaios.
}

1)as IX. Buch beginnt ngch einem kurzen, nus zwei parolistisch verwendeten Odysseeversen bestehenden Proömium ${ }^{1}$ ) nit lexikalischen Erörterungen des attizistisch orientierten Ulpian ilber die aufgctragrenen Speisen ${ }^{2}$ ). Die Speisenfolge ${ }^{3}$ ) weist Schinken mit pikanten Gewurzen und Saucen nuf, ist

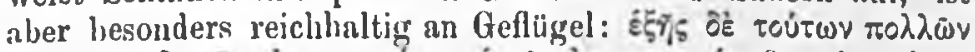

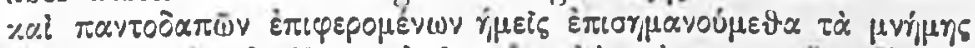

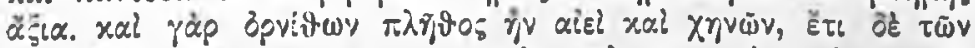

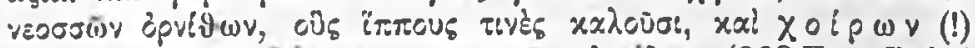

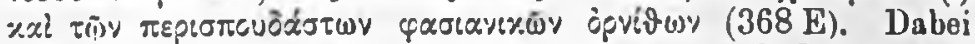
tiillit das Auftreten der Ferkel inmitten des Gefligels auf. O)ifenbar war das so ublich. Bevor aber nuu in eine IBesprechung dieses Themas eingetreten wird, milssen wir uns

8) Littmann stimmit mir brieflich zu.

9) Für den ersten hat schon Spiegelvergs Náchweis eines arabischen Buinortes des Kambyses dieso Grenze gezogen.

1) Vgl. K. Mengis, Die schriftstellerische 'l'echnik im Sophistenmahl des Athenaios. Studien zur (ieschichte und Kultur des Altertums X 5 , P'iderborn 1020, S. $76 \mathrm{f}$.

2) Ueber diese Art von Motivierung vgl. ebd. S. 66, $76 \mathrm{f}$.

3) Die Speisenfolgo ist offenbar die bei vornehmen Gastmäblern

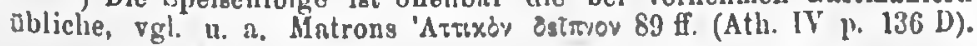


zunäclıst eine die Konposition störende linlago uber seliene Gemuse (369 A-372 $\mathrm{F}$ in Katalogform) gefullen lassen $\left.{ }^{4}\right)$. Mit szenischem Uebergang ${ }^{5}$ ) beginnt der Geflugelkatulog, dessen in Ganzen lexikalische Grundlage in unserer Ueberlieferung durch die den einzelnen Numıern vorgesetzten Lemmata noch besonders hervorgehoben wird ${ }^{6}$ ). Den Anfong des Katalogs: bildet eine Ablandlung uber die õpves im allgemeinen ( $373 \Lambda$ bis 374 D), wobei der Sprecher Myrtilos, ausgehend von einer Bemerkung uber das grammatische Geschlecht des Wortes

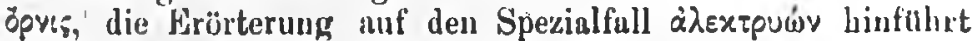
(373 E). Duran schlielit sich olne jede szenische Notjz oder

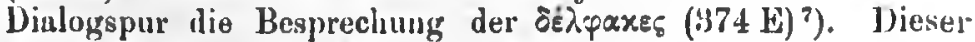
Abschnitt iibar das Schweinefleisch entwickelt sich zunächst. ganz in bisherigen kutalogartigen Rabmen, geht aber $376 \mathrm{C}$ (szenischer Uebergang!) in ziemlich lobhaften Dinlog aber ${ }^{8}$ ), der erst $384 \mathrm{~A}$ wieder dem Geflugelkatalog Platz machit. Auch hier herrscht während der ersten Nummern iler Dialog noch

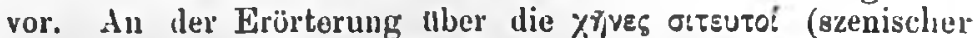
Uebergung) beteiligen sich Ulpian, Plutarch, Myrtilos und Kynulkos. Auch zu diesem Gang werden I)elikatessen ser-

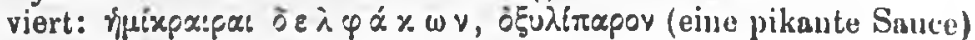
u. a. Die Erörterung scheint so lebhaft greworden zu sein, daL: Ulpian und Myrtilos den nächsten Gung, den als Delikatesse besonders geschütaten Fasan, rerpatit liaben (386 D f.). Auf Ulpians Bitte hält Myrtilos uber den Fasan einen mit zahlreichen Zitaten aus Aristophanes, Theophrast, Aristoteles u. n. gespickten Vortrag. In folgenden ist jede Dialogspur getilgt. Das ganze Stuck 387 IF-398 B unterscheidet sich von den lexikalischen Partien sowohl hinsichtlich seiner ganzen Anlage"), als auch hinsichtlich der breiten Ausfuhrlichkeit in der Beschreibung des Geflngels. Als Quelle dieses zusammenhüngenden, einheitlichen Abschnittes hat Wellmam dus Werk Tsp? $\zeta$ फ़v des \%oologen Alexamdros von Myndos ermittelt ${ }^{10}$ ). Nach

") Eine der Stellen, die far die Beurteilung der Schriftstellerei des Athenaios ron Bedeutung sind. Vgl. Die schriftstellerischo Technik และ. S. $5 \mathrm{ft}$.

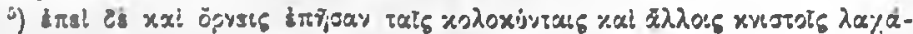
vo:5 $x+\lambda$.

6) Als bexeichnendes Gegenstuck vgl, den Fischkatillog des VII. Buches und dazu "Dis schriftstellerische 'Technik" usw. $74 \mathrm{f}$.

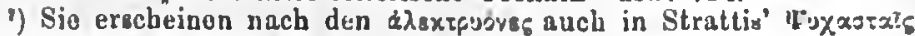
(I $728 \mathrm{~K}$. Ath. IX $373 \mathrm{f}$. Stichwort?).

s) Daraber vgl. "Die schriftstellerische Technik" \$. 20. 4.

9) So fehlt z. B. die dort abliche alphubetische Anordnung wio 2. B. im Fischkistalog des VIf. Gerude áer mibjglickto Versuch des Athenaion, sein Material alphabetisch 24 ordnen (388 B ff.) beweist, dali hier nicht mit oiner lexikalischen Quelle zu rechnen ist.

in) Hermes XXVI (1891). 
diesem Gewährsmamn also bespricht Athenaios in diesem $\mathrm{Ab}$ -

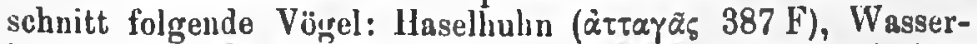

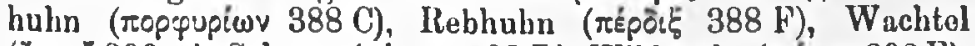

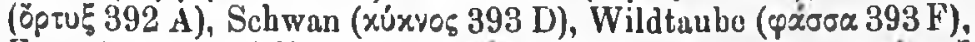

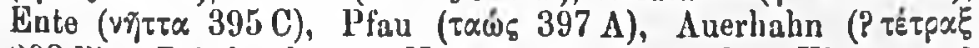
398 B). Bei der letzten Nummer setzt zwischen Ulpian und Larensis der Dialog wieder ein (398 B). Innerhalb dieses in sich geschlossenen Abschnitts lassen sich folgende lexikalischen Einlagen feststellen: $388 \mathrm{BE}-389 \mathrm{~A}, 390 \mathrm{~A}, 391 \mathrm{D}, 392 \mathrm{ABEF}$, $393 \mathrm{BC}, 394 \mathrm{~A}, 395 \mathrm{BC}, 395 \mathrm{~F}-397 \mathrm{~A}$. Mit Ausnahme der letzten ( $395 \mathrm{~F}-397 \mathrm{~A}$ ), die in szenischer Motivierung einige pikante Fleischspeisen (dabei auch dio unvermeidlichen Spanferkel $396 \mathrm{C}$ ) zur Diskussion stellt, handelt es sich durchweg. um Erginzung des wissenschaftlichen zoologischen Materials durch Dichterzitate (meist aus der Komödie) nach der lexikalischen Seite hin. Dali diese Exzerpte Beruhrungen mit Hesych aufweisen, scheint, olme daß es zwingend bewiesen werden könnte, auf den p. $387 \mathrm{D}$ zitierten Pamphilos als Gewährsmamn hinzuweisen, auf dessen Sammelwerk auch die ubrigen lexikalischen Ausfuhrungen dieses Buches zu fußen scheinen ${ }^{11}$ ).

Das Deipnon, uber das in den ersten Buchern referiert wird, geht IX $408 \mathrm{~B}$ zu Ende: es wird Wasser zum Reinigren der Flinde gebracht. $\Lambda$ uf das X. Buch fuillt der offizielle Potos (X 422 E) ${ }^{12}$ ). Mit Buch XI setzt ein newer Tug ein :

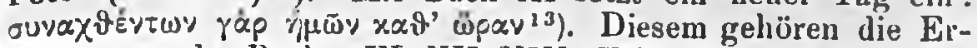
örterungen der Bucher XI, XII, XIII, XIV. Das Gelage dieses 'Tages findet XIV $664 \mathrm{~F}$ seinen $\mathrm{Abschlul}$. Mit diesen unzweideutigen Daten steht in unertrïglichem Widerspruch, dals das Werk in seiner heutigen Gestalt otfenbar Anspruch crhebt, Darstellung eines Mustergastmabls zu sein. Ich habe früher an anderer Stelle gezeigt, daf , diese Absicht der ersten Konzeption jedenfalls noch fremd "war ${ }^{14}$ ). Eine weitere Stitze erhalten jese Darlegungen durch die Untersucliungen, die ich heute vorlegen kamn.

Das XIV. Buch befabt sich mit der Unterhaltung del Zechenden. Sis wird bestritten von Akrobaten und Musikanten. Naturlich schliebt sich an die Darbietungen der Kunstler und ihre Instrumente eine breite Diskussion an. Wälırend eines Vortrags des Masurios werden die sog. ठEútefas.

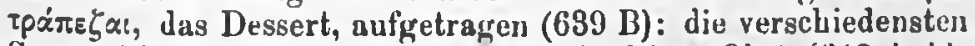
Sorten Kuchen (Katalog der Kuchen), feines Obst (643 A bis ${ }^{11}$ Vgl. K. Bapp, Ijeitrilge zur Quellenkritik des Athen us, Comment. Ribbeck. Lps. 1888, S. 251 ff.

12) Ygl. ${ }_{10}$ Die schriftetellerische Technik * uaw. S. $12 \mathrm{f}$.

19) Ueber die Zeitverhilltnisse vgl. ebd. S. 4.

14) Ebd. \&. $5 \mathrm{fr}$. 
653 D) und liäse. Der schriftstellerischen 'T'echuik des Athennios gemüb tritt man zuerst in eine allgemeine Hrörterung

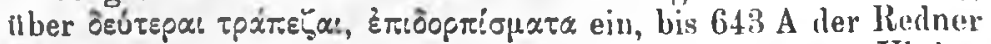
Pontianos zu den einzelnen Leckerbissen ubergeht. Ulpian

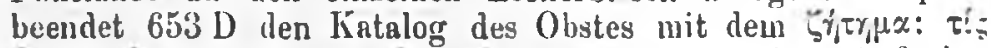

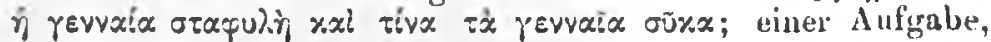

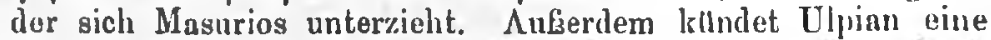

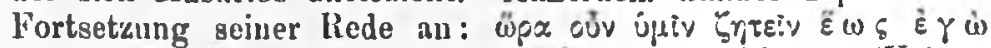

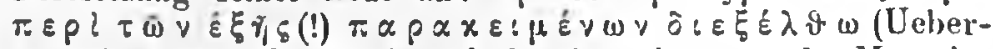
uang!). $\Lambda$ ber als $654 \mathrm{~A}$ nach den $\Lambda$ usfitirungen des Masurios Ulpian, wio es nach dem Voraufgegungenen wahrscheinlich ist, wiederbeginnt, ist der alte Uebergang vergessen, ein neuer, In dio Situation gar nicht passender tritt an seine Stelle:

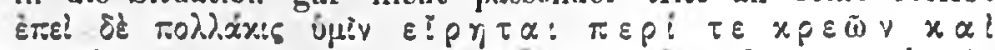

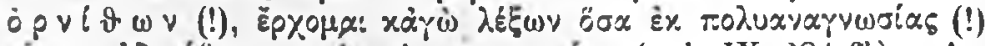

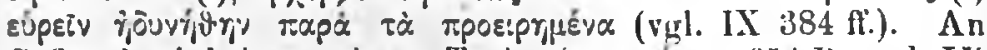

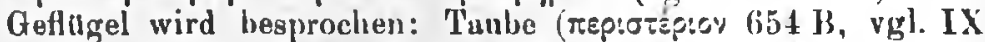

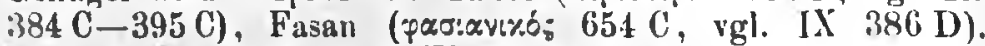

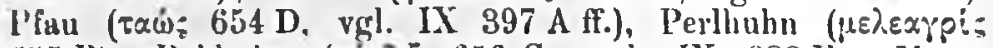

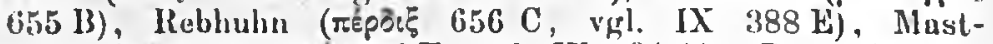

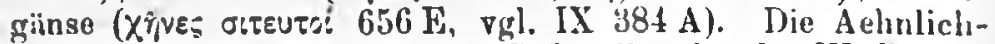
keit dieses Abschnittes mit dem Gefligelkatalog des IX. Buches ist frappierend, sie geht sogar soweit, daf an beiden Stellen dio Aufzählung des Geflugels unterbrochen oder ergänzt wird von Berwerkungen über Schweinefleisch (IX $396 \mathrm{C}$, X II $655 \mathrm{~F}$. 656 F), Euter (oṽ $\alpha \rho$ IX 399 C, XIV 656 E) und Hasen ( $\lambda \alpha \gamma(\omega)$ IX 399 D, XIV 656 C). Vielleicht war der Geflugelkatalog des XIV. Buches urspranglich noch umfissender oder wenigsténs umfassender beabsichtigt, als er uns liente vorliegt. Darauf kann die Bemerkung denten, die Ulpian XIV 652 F mitten im Obstkatalog im Anschluli an ein Phoinikidescitat

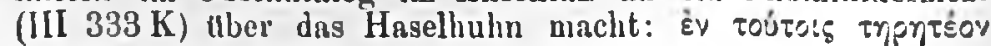

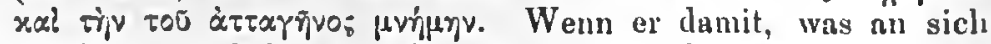
durchaus möglich ist, nicht uuf den I. Geflugelkatalog Bezug nimmt, so lüitten wir darin eine Vorbereitung des II. Katalogs zu erblicken. In diesem sucht man aber dis Haselhuh vargoblich.

Es fragt sich nun, wie diese erneute Behandlıng des GeHilgels und der Fleischispeisen an dieser Stelle zu erliliiren ist. Dafi der Platz für dio Erörterung der denkbar schlechitestre ist, wird jedermann zugeben. Denn es ist schlechterdings nicht einzusehen, was Geflügel und Fleischspeisen mitten unter lem Dessert zu suchen haben. Dazu kommt, dak in der heutigen Fassung die Erörterung dieser Dinge so gut wie unmotiviert einsetzt. Die Schwierigkeit lüst sich aber mit einem 
Mal, wenn wit uns zu der Anunhme verstehen, datis das heutige Mustergastmahl nicht den ursprünglichen Zustand darstellt. IVir werden vielmehr in dem II. Geflügelkatalog die Trilmmer eines ursprunglich selbstindigen Deipnons zu erblicken haben, das nachträglich einem sekundären Kompositionsprinzip geopfert wurde. Darum auch die selbst bei Athonaios so besonders ungeschickte Art der Motivierung (XIV 65 \& A, cap. 69) an Stelle der ursprïnglich szenischen ${ }^{15}$ ).

Nun erhebt sich aber die l'rage, ob Athenaios bei dieser Joppelbehandlung desselben Stoffes ein und derselben Quelle refolgt ist, oder ob er, was von vornherein nicht abzuweisen sein wird, anf verschicdenen Grundlagen nufbaut. Zur Beantwortung ist eine Gegenuberstellung der parallelen Abschnitte notwendig.

\section{$\mathrm{x}$}

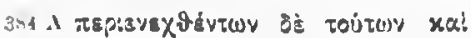

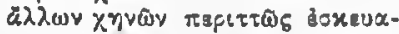

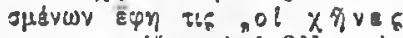

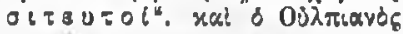

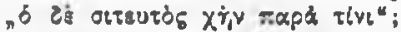

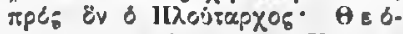

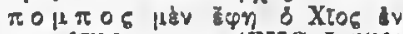

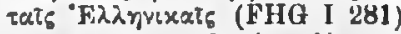

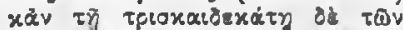

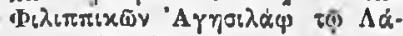
"wvi ale Alyustov deptxopesve

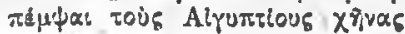

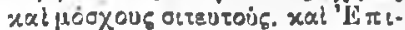

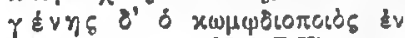
Baxxu: ६ทvlv (II $417 \mathrm{~K}$ ) - -

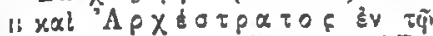

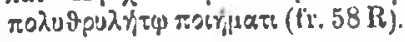

\section{XIV.}

057 II Xทvอั

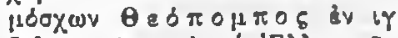
एะ่ (FHG I 297 u. 281), है ols t $\mu \varphi \alpha$ -

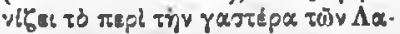

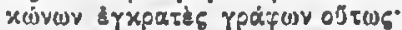

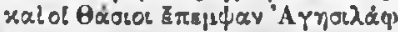

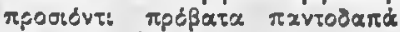

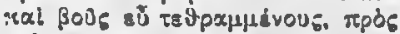

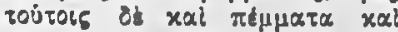

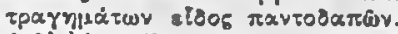

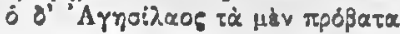

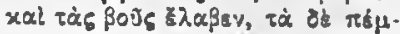

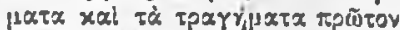

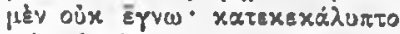

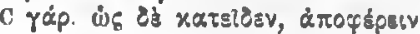

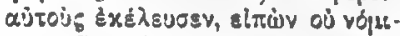

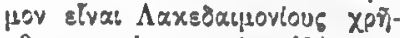

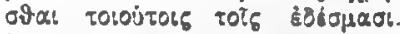

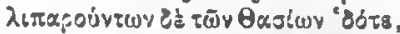

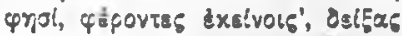

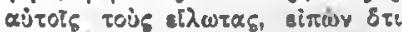

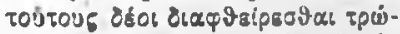

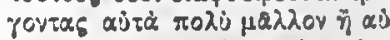

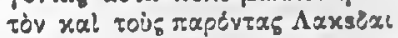
poviwe'.

Daran schliefit mit szenischer Motivierung in beiden Füllen ein aus Schweinefleisch bestehender Speisegung: IX $384 \mathrm{C}$ :

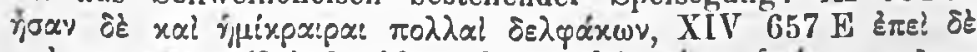

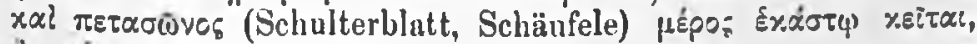

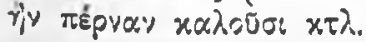

15) Vgl, XIV $657 \mathrm{H}$. 
s\&8 I) Im kifer der Unterhaltung haben Ulpian und Myrtilos den Fasan an sich voruber. gehen lassen. Ulpian verspricht dein Myrtilos, ihn fur den entgangenen Leckerbisen zu entachadigen, fulls er sich $z u$ einem Vortritg aber den Fasan herbeilasse. Myrtilos willfuhrt und gibt folgende Belege :

"A ple b c iv Tuvalxopariq (II $238 \mathrm{~K}$ )

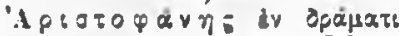
Opvio:s in) (v.67 f.)iv Neçt2a:s (v. 109)

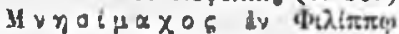
(III $442 \mathrm{~K}$ )

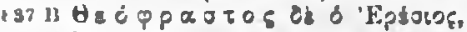

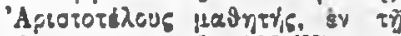

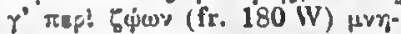

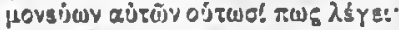

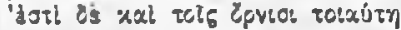

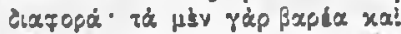

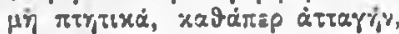

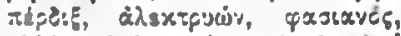

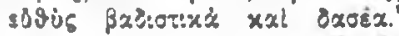

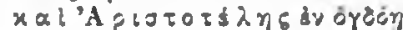
Givew iтtopias yedze: titz

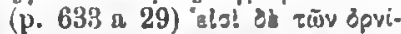

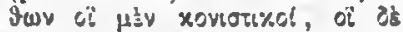

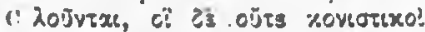

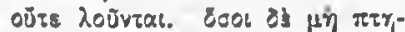

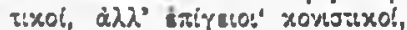

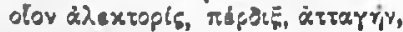

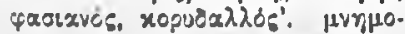

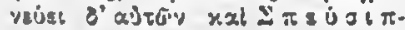
$\pi 05$ iv beutiepe "Opolwy. -

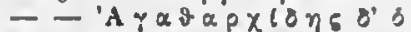

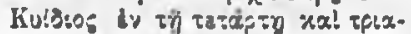

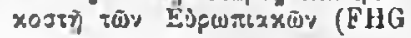

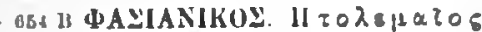

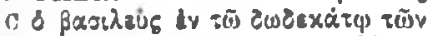

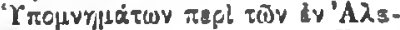

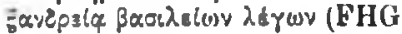

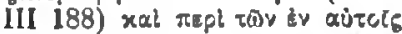

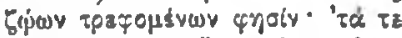

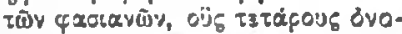

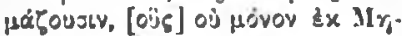

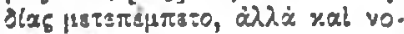

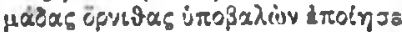

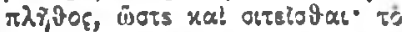

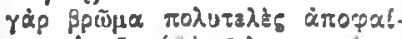

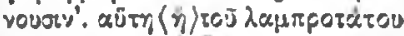

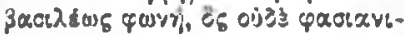

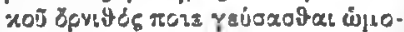

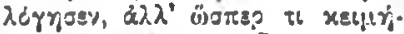

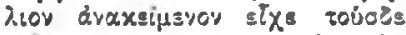

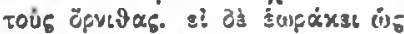

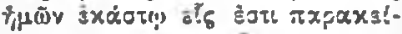

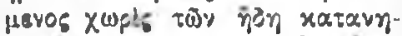

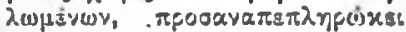

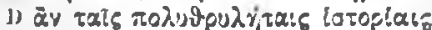

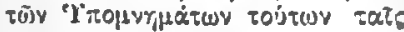

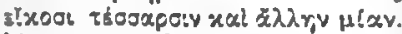

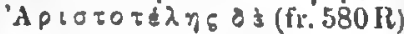

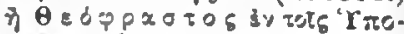

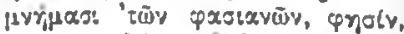

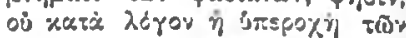

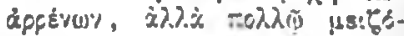
$\left.v(s) y^{17}\right)$.

18) Die ausfuhrlicho Inhaltsangabe der Szene aus den aristoplanischen Vogeln durch Ath. IX $3 \& 6 \mathrm{~F}$ steht in schilrfotem Gegeneatz zu der sonstigen Zitierwcise. Sio fält ans der streng lexikalischen Umgobung heraus.

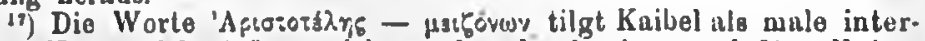
iecta Vermutlich stülit er sich an der durch sie veranlafiten Unterbrechung der Bemerkungen Ulpians aber Ptolemaios. Ulpian fuhrt

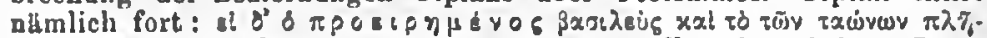
Sos twpazx: $x \tau \lambda$. Indes iut Knibela Anetó m. E. unberechtigt. Denn ganz abgesehon davon, das er an die Kompositionstechnik des Athenuios selbat damit einen zu ccharfen Mafastab anlegt, beweist gerade

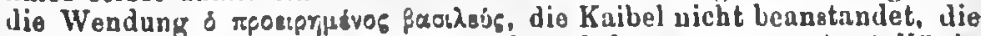
Eichtheit des Eingchubs. Der Verwein unf den pvorgenannten" Konig whre nicht nur nicht notig, Bondern auch unlogiech, wenn die beideu Stacke so lackenlos zusammenschliefen wurden, wis Kitibel es will. 
I) III 194) - - K $\alpha \lambda \lambda 6 \xi \mathrm{s}$.

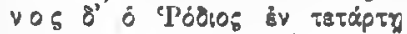

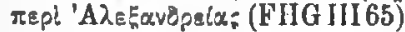
- - Ap T 14 lo wpos ós

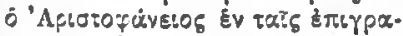

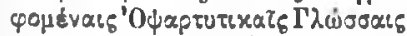

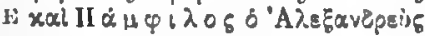

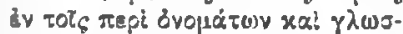

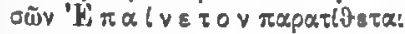
$\lambda$ irovte $x \tau \lambda$. II $\tau 0 \lambda \in \mu \alpha \tau 05$

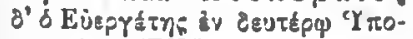

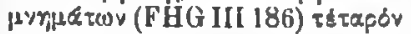

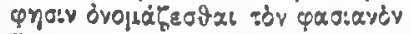
Epviv.

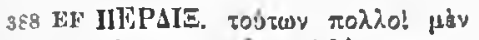

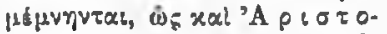

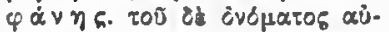

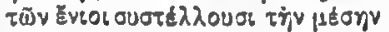

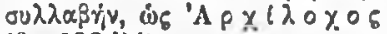
(fr. $106 \mathrm{~B}^{4}$ ) -

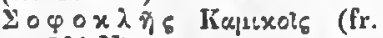
$301 \mathrm{~N})$ - -

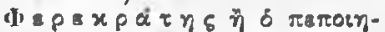

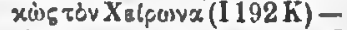

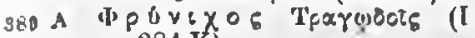
$384 \mathrm{~K})$ - -

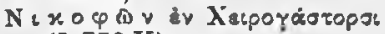
(I $779 \mathrm{~K}$ ) -

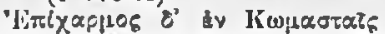

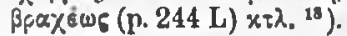

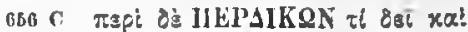

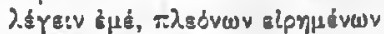

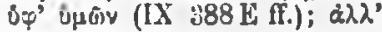

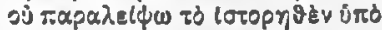
'H $\gamma \eta \sigma \alpha v \varepsilon \rho O u$ iv rols ' $1 \pi 0$ -

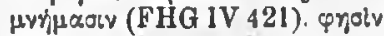

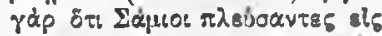

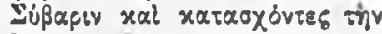

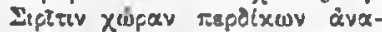

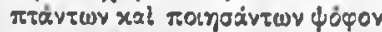

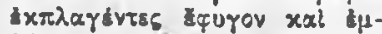

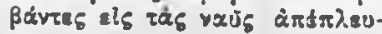
axv.

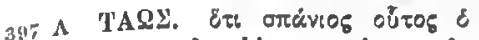

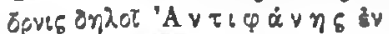

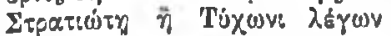
cítws (II $99 \mathrm{~K}$ )

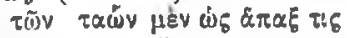

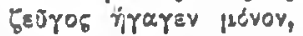

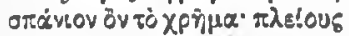

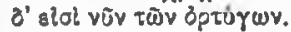

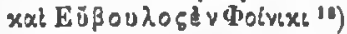

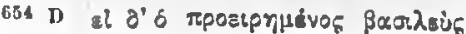

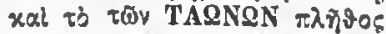

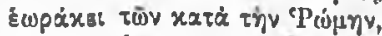

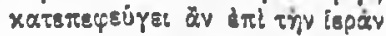

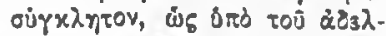

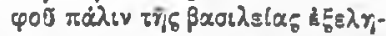

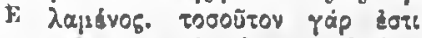

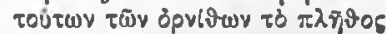

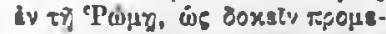

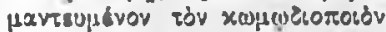

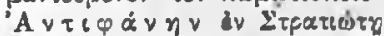

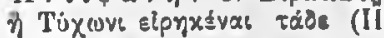
$99 \mathrm{~K})^{\text {? }}$

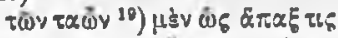

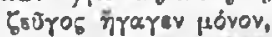

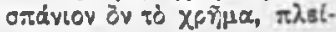

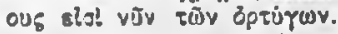

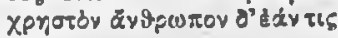

18) Die anschliefeuden zoologisahen Belege stammen aus einer ganz anderen Quelle, nämlich aus dem Werk des Zoologen Alerander von Myndos. Vgl. Wellmann a. a. $O$.

19) тbv cxiv $A F$, cf. IX $397 \AA$. Die Korraptel entatand aus einem Ilorfehler beim Diktat. 


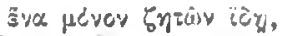

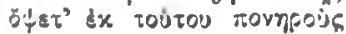

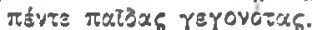

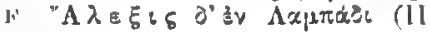 $340 \mathrm{~K})--$

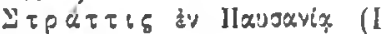 $718 \mathrm{~K})$ - - 'A $\vee \alpha \xi \alpha \vee \delta p l-$ ठ $\eta \varepsilon$ है $\mathrm{Me} \lambda \epsilon \lambda(\dot{\omega} \tau)$ (II $145 \mathrm{~K}$ )

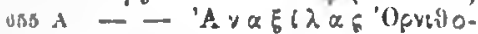

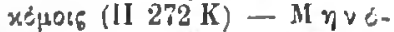 ว O โ O

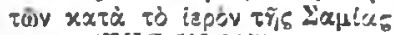 "IPses (FHG HII 105) - - -

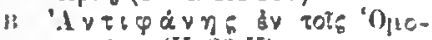 Txtelets (II $83 \mathrm{~K}$ ).}

$309 \mathrm{C}$ or Iteppots (I $217 \mathrm{~K}$ )

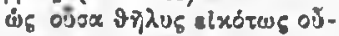
जas ६сорй.

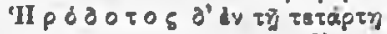

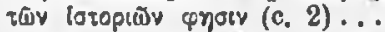

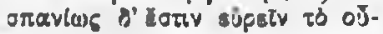

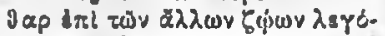

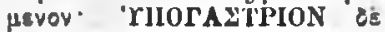

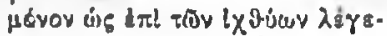

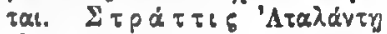
(I $713 \mathrm{~K})-\mathrm{A}$ - $\delta \pi \circ \mu$ $\pi \circ 5 \mathrm{~K} \alpha \lambda \lambda \alpha$ lo\%pep (I $738 \mathrm{~K}$ ) $x \tau \lambda$.

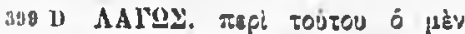

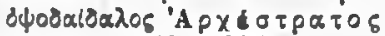

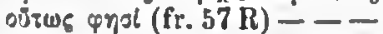

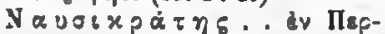
oles. . . . (II $296 \mathrm{~K}$ ) - - 'A $\lambda \times \propto$ เ० (I $759 \mathrm{~K}$ ) $x \tau \lambda$.
OrAATOL of T $\eta \lambda=x \lambda=$

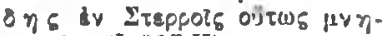
म10yses (I $217 \mathrm{~K}$ ).

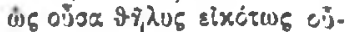

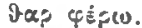

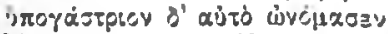

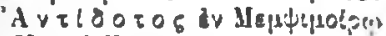
(II $410 \mathrm{~K}$ ).

Die Belege sind bei diesem Punkt völlig verschieden.

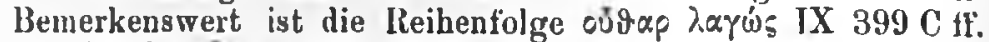
und $\lambda x \gamma \omega \dot{s}$ oủvap XIV $656 \mathrm{C}-\mathrm{E}$.

Was lehrt diese Gegenulberstellung? Hat Athenaios beiden Katrlogen im allgemeinen die gleiche Quelle zugrunde gelegt - nelımen wir an das Lexikon des Pamphilos -, so müssen wir uns, um die Verschiedenheit ler Belege zu erk]ären, zu der Vermutung entschlieken, der Schriftsteller habe das Materinl von vornherein auf die beiden Deipna so verteilt, dab die späteren Zitate die ersten ergïhzen mussen. 1)ass wilrde uberall stimmen, nur bestïnde dann XIV 654E die Eirgänzung in der Erweiterung des IX $397 \mathrm{~A}$ zitierten Antiphanesfragments (II $99 \mathrm{~K}$ ) um zwei weitere Verse, wiihrend das 
Telekleideszitat (1 217 K) XIV $656 \mathrm{E}$ eine vollkommene Dublette zu IX $399 \mathrm{C}$ ist. Das mufs gegen eine solche Annahne hedenklich stimmen. $\Lambda$ ber auch abgesehen davon ist eine derartige Kompositionstechnik fur jeden, der die schriftstellerische Art des Athenaios kennt, durchnus unwahrscheinlich. Dann bleibt nur anzunehmen, dn is Athenaios die Zitate des XIV. Buches allerdings als Ergänzung der Angaben des JX. Buches angeschen wissen wollte. Wozu sonst der ausdrilckliche Hinweis anf die fruhhere Errörterung XIV 654 A ? Daf er dio $\mathrm{Be}-$ leue uber offenbar anderen Quellen verdankt, wie die des IX. Buches, darüber können uns vielleicht die Fragmente selbst und die Zitierweize des Athenaios Aufschluli geben.

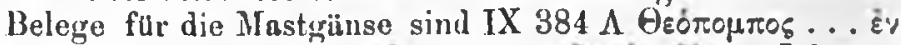

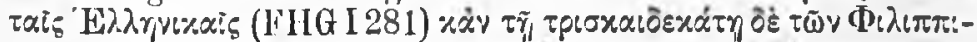

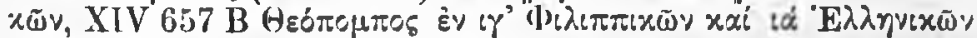
(FHG 297 แ. 281). IX 381 ist die Einkleidung lebhaft dialogisch, dio zweite Stelle ist ein Stuck Lehrvortrag Ulpians, wobei das

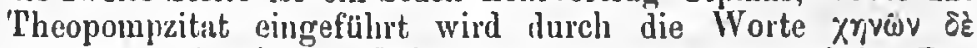

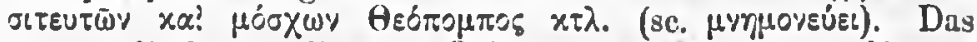

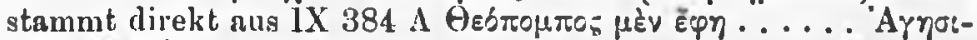

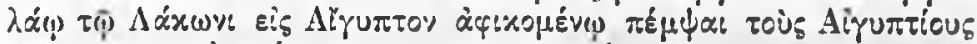

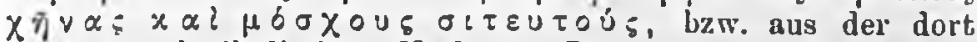
beniitzten lexikalischen Vorlage. Die zweite Stelle bezielit sich nlso ausdrucklich auf die erste. Der Unterschied der beiden Stellen besteht darin, dals die erste eine knappe Inhaltsangabe des Theopompstückes gibt, wïhrend die zweite es ausschreibt, wie IX $387 \mathrm{E}$ sich gegenüber dem ausfuhrlichen Vitat aus den Hypomnenata des Ptolemaios Euergetes XIV 654 B auf einen kurzen Hinweis beschränkt. Nun ist aber in dem XIV 657 B ausgeschriebenen Theopompzitat von Gänsen uber-

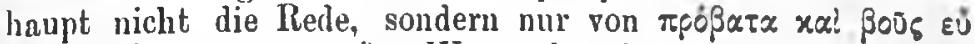

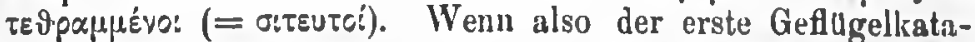
log wirklich im ganzen auf eine einheitliche lexikalische Quelle zuriickzuführen ist, so können für eine solche nur Stlicke in Betracht kommen, die sich in den gesteckten Rahmen fugen. Das kanu aber von dem Theoponpzitat des XIV. Buches nicht gesagt werden. Demnach haben wir es entweder überhaupt mit zwei verschiedenen Zitaten zu tun, was nach der Zitierweise möglich ist, obwohl der Inhalt $2 u$ widersprechen scheint; oder die lexikalische Notiz des IX. Buches entstand durch eine Ungenauigkeit des Sammlers. Auch für das Zitat des XIV. Buches ergibt sich eine Alternative: entweder hat sich Athenaios, der den Theopomp selbst gelesen und exzerpiert ${ }^{20}$ ) und demnach offenbar das Zitat aus dem eigenen Zettelkasten bei-

20) Vgr. Wentzel, Pauly-Wissowa RE. Il 2032. 
gesteuert hat, bei der Identifizierung geirrt. Dem steht aber wieder der sonst ganz abereinstimmende Inlaalt entgegen. Oder er wollte an der ungenauen Zitierweise seines Gewiihrsmanns positive Kritik uben.

Lehrreich ist much die Art und Weise, wie XIV 65+ D

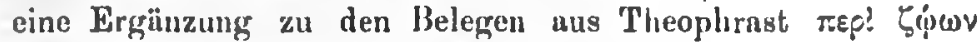
(fr. $180 \mathrm{~W}$ ) und Aristoteles Tiorgeschichte (p. 633 a 29) ge-

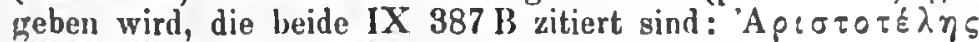
ô है

Einheitlich in ihrer Art sind die lexikalischen Belege für das Rebhuhn IX $388 \mathrm{~F}-389 \mathrm{~A}$, wo die Exzerpte aus dem Werke des Zoologen Alexander von Myndos einsetzen. Sie werden XIV $650 \mathrm{C}$ lediglich um ein Hegesanderzitat (FIIG

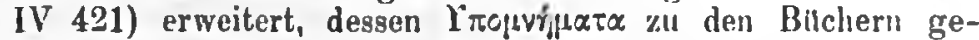
hörten, dio Athenaios am allermeisten nusschrieb ${ }^{22}$ ).

Fïr den P'fau werden, abgesehen von den Belegen, die wohl sicher aus dem Buche des Alexander von Myndos stanmen, mit einer Ausnahme nur Konikerzitate beigebracht, von denen der grögere Teil auf den zweiten Katulog fïllt. Unter diesen Zitaten kehrt das schon IX 397 A angefuhrte $\Lambda$ ntiphanesfrngment (II 99 K) um zwei Verse erweitert XIV 654 D wieder. Das beweist einmal, dub die ganze Zitatenreihe der zweiten Stelle die der ersten ergänzen soll. Dann geht daraus bllndig hervor, dak mindestens das erweiterte Antiphnneszitat nicht derselben Mittelquelle entstammt wie das gekürte des IX. Buches. Denn die beiden neuen Verse haben mit dem eigentlichen

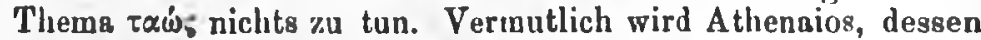
Spezialgebiet ja gerade die Komödie war ${ }^{27}$ ), die Verse aus seinem eigenen Exzerptenmaterial beigesteuert haben.

Fur die Entscheidung der Frage, ob an beiden Stellen dieselbe lexikalische Quelle vorliegt, sind die Nummern củsap und $\lambda x y$ ws unergiebig, weil sie zwar bei Athenaios im Ruhmen des Deipnons als Beilagen zum Geflugel ihren Platz haben, in einen reinen Geflugelkatalog jedoch, wie wir ihn für eine lexikalische Vorluge voraussetzen mussen, nicht pissen. Indes sind gerade sie für die Arbeitsweise des Athenaios bezeichnend. Die Belege entstammen mit zwei Ausnahmen der Komödie, also vermutlich dem Zettelkasten des Athenaios selbst. Den Ilerodot, den er zu IX $399 \mathrm{C}$ anfuhrt, hat er ebenfalls im Original studiert und exzerpiert. Charakteristisch ist die Du-

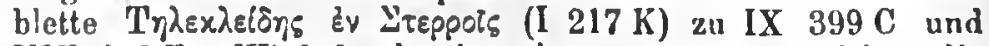
XIV $656 \mathrm{E}$. Wird durch sie schon so gut wie sicher die pixdé.

21) So zitiert er aberall die pseudoariatotelischen '

i) Wontzel a. a. 0.

2a) Ath. VIII $336 \mathrm{D}$. 
Möglichkeit ausgeschlossen, dafi das Material ein und derselben Vorlage mechanisch auf zwei verschiedene Stellen verteilt wurde, - ich glaube das hiebe in diesem krassen Fall selbst einem Athenaios Unrecht tun - so gewährt sie uns daraber hinaus noch einen Einblick in die 'T'echnik des Sch r if tstellors $\Lambda$ thenaios. Gewis wird man die Variante yop $\varphi s \omega^{\prime} \omega$ in den Telekleideszitat ebenso leicht paläographisch wie als Hörfehler beim Diktat erklüren können 24). Gliicklicherweise hat sich aber Kaibel ror der öden Gleichmacherei geliutet, die in solchen fiallen nur zu schmell bei der Hand \%u sein pflegt, um die richtige Ueberlieferung zu verderhen. In unserm Fall kann die Diskrepanz eine gewollte sein. Möglich, dab A thenaios beide Lesarten vorfand und an der zweiten Stelle Kritik an der ersten Lesart (lben wollte ${ }^{25}$ ).

Dns IInuptergebnis: Athenaios hat im IX. und XIV. Buch je einen durch jeweils gleichartige Fleischspeisen erweiterten Geflikglkatalog eingelegt. Während der des IX. Buches an seiner Stelle wohl am Platz ist, stört der des XIV. Buches

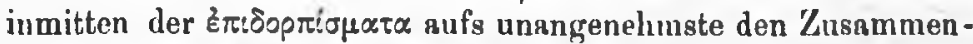
hang. Das war nicht der Fall, wenn dieser Katslog ursprünglich analog dem des IX. Buches einem besonderen Deipnon angehörte. Wir haben hier also wieder eine Spur zum mindesten eines fruheren Entwurfes des Sophistenmabls, vielleicht sber auch darüber hinaus einen Beweis für die 'I'rummerhattigkeit unserer Ueberlieferung.

Der 7weite Katalog gibt sich ausdrijcklich a!̣ Ergänzung res ersten. Aber wïhrend dieser mit grober Walirscheinlichkeit, wenigstens was den rein lexikalischen 'leil angeht, nuf ciner entsprechenden Vorlage, vermutlich dem Luexikon des Pamphilos, basiert. ist das bei dem zweiten nicht der Fall. Die Auswahl der Fragmente und die Art ihrer 7itierung läl die Vermutung begründet erscheinen, dak hier Athennios das im IX. Buch gegebene Material seines Gewährsmanns durch eigene selbständige Exzerpte ergünzt hat.

Freiburg i. Br.

Karl Mengis.

\section{Zur Aetna V. 63.}

Die viel besprochene Stelle - sie betrifft den Gigantenkampf - bedarf bloki der Einfügung eines l3uchstrbens:

2) Vgl. Kurt Zepernik, Die Exzerpte des Athenas in d. Deipno. sophisten u. ihre Glaubwardigkeit, Philol. N. F. XXXI (1921), S. \$i29.

${ }^{25)}$ copalv ist in solchen Füllon das gebriuchlichere Verbum. Vgl. Lobeck, Phrynichus 585 .

Phllologas T, XXVIII (N. F, XXXII), s!!. 